Tôhoku Math. Journ.

21(1969), 523-531.

\title{
ON THE ABSOLUTE SUMMABILITY OF FOURIER SERIES
}

\author{
ISTVÁN SZALAY
}

(Received September 17, 1968)

1. Introduction. Let

(1)

$$
f(x) \sim \frac{a_{0}}{2}+\sum_{n=1}^{\infty}\left(a_{n} \cos n x+b_{n} \sin n x\right)=\sum_{n=0}^{\infty} A_{n}(x)
$$

and let $s_{n}(x)$ and $\sigma_{n}^{\alpha}(x)(\alpha>-1)$ denote the $n$-th partial sum and $n$-th $(C, \alpha)$ mean of Fourier series (1), respectively. If the series

$$
\sum_{n=0}^{\infty}\left|\sigma_{n}^{n}(x)-\sigma_{n-1}^{n}(x)\right|
$$

is convergent, we say that the series (1) is absolutely summable $(C, \alpha)$ or summable $|C, \alpha|$ at the point $x$.

We have

$$
\left\{\sigma_{n}^{\alpha}(x)-\sigma_{n-1}^{\alpha}(x)\right\}=\frac{\tau_{n}^{\alpha}(x)}{n}
$$

where

$$
\boldsymbol{\tau}_{n}^{\alpha}(x)=\frac{1}{A_{n}^{\alpha}} \sum_{k=1}^{n} A_{n-k}^{\alpha-1} k A_{k}(x)
$$

and

$$
A_{n}^{\alpha}=\left(\begin{array}{c}
n+\alpha \\
n
\end{array}\right)
$$

For $f(x) \in L^{p}(1 \leqq p<\infty)$ we define

$$
\omega_{p}^{(1)}(t, f)=\sup _{0<h<l}\left\{\int_{-\pi}^{\pi}|f(x+h)-f(x-h)|^{p} d x\right\}^{1 / p}
$$


and

$$
\omega_{p}^{(2)}(t, f)=\sup _{0<h<t}\left\{\int_{-\pi}^{\pi}|f(x+h)+f(x-h)-2 f(x)|^{p} d x\right\}^{1 / p}
$$

SUNOUCHI [7] proved the following theorems.

THEOREM A. Let $1<p \leqq 2$. If

$$
\omega_{p}^{(1)}(t, f)=O\left\{\left(\log \frac{1}{t}\right)^{-\frac{1}{2}-\delta}\right\} \quad(\delta>0),
$$

then the series (1) is summable $|C, \alpha|$ almost everywhere for $\alpha>1 / p$.

THEOREM B. Let $1<p \leqq 2$. If

$$
\omega_{p}^{(1)}(t, f)=O\left\{\left(\log \frac{1}{t}\right)^{-\left(1-\frac{1}{p}+\frac{1}{2}+\hat{o}\right)}\right\} \quad(\delta>0),
$$

then the series (1) is summable $|C, 1 / p|$ almost everywhere.

We prove the following theorems which generalize SUNOUCHI's theorems.

THEOREM I. Let $f(x) \in L^{p}(1<p \leqq 2)$ and let $\left\{\mu_{n}\right\} \quad(n=1,2,3, \cdots)$ be a monotonic non-increasing sequence tending to zero, and satisfying the condition

$$
\sum_{n=1}^{\infty} \frac{1}{n\left(\sum^{n} \mu_{k}\right)^{2}}<\infty
$$

If

$$
\sum_{n=1}^{\infty} \mu_{n} \omega_{p}^{(2)}\left(\frac{1}{n}, f\right)<\infty
$$

then the series (1) is summable $|C, \alpha|$ almost everywhere for $\alpha>1 / p$.

THEOREM II. Let $f(x) \in L^{p}(1<p \leqq 2)$ and let $\left\{\rho_{n}\right\}(n=1,2, \cdots)$ be a monotonic non-decreasing sequence such that $\rho_{n}[\log (n+1)]^{-1 / 2}$ is non-increasing and satisfying the condition: 


$$
\sum_{n=1}^{\infty} \frac{1}{n \rho_{n}^{2} \log (n+1)}<\infty
$$

If

$$
\sum_{n=1}^{\infty} \frac{\rho_{n} \omega_{p}^{(2)}\left(\frac{1}{n}, f\right)}{n[\log (n+1)]^{\frac{1}{p}-\frac{1}{2}}}<\infty
$$

then the Fourier series of $f(x)$ is $|C, 1 / p|$ summable almost everywhere.

Using an equivalence theorem of LEINDLER [6] (Satz III), we get from Theorem I and Theorem II the following corollaries.

Corollary I. Let $f(x) \in L^{p}(1<p \leqq 2)$ and let $\mu(x)(x \geqq 1)$ be a nonincreasing function. If $\mu_{n}=\mu(n)$ satisfies the condition (2) and if for $a$ certain $\beta(>0)[\log (x+1)]^{-\gamma_{1}} \geqq x^{\beta} \mu(x) \geqq[\log (x+1)]^{-\gamma_{2}}\left(\gamma_{1}<\gamma_{2}\right)$, then both conditions

$$
\int_{0}^{1} \frac{\mu\left(\frac{1}{t}\right)}{t^{2}}\left(\int_{0}^{2 \pi}|f(x+2 t)+f(x-2 t)-2 f(x)|^{p} d x\right)^{1 / p} d t<\infty
$$

and

$$
\sum_{n=1}^{\infty} \mu \cdot(n) E_{n}(f, p)<\infty^{1)}
$$

are sufficient for the summability. $|C, \alpha|(\alpha>1 / p)$ of the Fourier series (1) almost everywhere.

COROLLARY II. Let $f(x) \in L^{p} \quad(1<p \leqq 2)$ and let $\rho(x)(x \geqq 1)$ be a nondecreasing function. If $\rho_{n}=\rho(n)$ satisfies the condition (4) and if for a certain $\alpha(>0)$

$$
[\log (x+1)]^{\frac{1}{p}-\frac{1}{2}-\gamma_{1}} \geqq x^{\alpha-1} \rho(x) \geqq[\log (x+1)]^{\frac{1}{p}-\frac{1}{2}-\gamma_{2}}
$$

$\left(\gamma_{1}<\gamma_{2}\right)$, furthermore if $\rho(n)[\log (n+1)]^{-\frac{1}{2}}$ is nonincreasing, then both conditions

$$
\int_{0}^{1} \frac{\left.\rho_{1}^{(} \frac{1}{t}\right)}{t|\log t|^{\frac{1}{p}-\frac{1}{2}}}\left(\int_{0}^{2 \pi}|f(x+2 t)+f(x-2 t)-2 f(x)|^{p} d x\right)^{1 / p} d t<\infty
$$

1) $E_{n}(f, p)$ denotes the best approximation of $f(x)$, in the sense of the metric of $L^{n}$, by trigonometric polynomials of order $(n-1)$. 
and

$$
\sum_{n=1}^{\infty} \frac{\rho(n) E_{n}(f, p)}{n[\log (n+1)]^{\frac{1}{p}-\frac{1}{2}}}<\infty
$$

are sufficient for the summability $|C, 1 / p|$ of the Fourier series (1) almost everywhere.

It is easy to see that in the case

$$
\mu_{n}=n^{-1}[\log (n+1)]^{\varepsilon-\frac{1}{2}} \quad\left(0<\varepsilon<\min \left(\frac{1}{2}, \delta\right)\right)
$$

Theorem I includes Theorem $\mathrm{A}$ and if

$$
\rho_{n}=[\log (n+1)]^{\varepsilon} \quad\left(0<\varepsilon<\frac{1}{2}\right)
$$

then Theorem II implies Theorem B.

It is also easy to verify that if

$$
\mu_{n}=\frac{[\log \log (n+2)]^{1 / 2+\varepsilon}}{n[\log (n+1)]^{1 / 2}} \quad(\varepsilon>0)
$$

and

$$
\omega_{p}^{(2)}(t, f)=O\left\{\left(\log \frac{1}{t}\right)^{-1 / 2}\left(\log \log \frac{1}{t}\right)^{-3 / 2-\hat{-}}\right\} \quad\left(0<\varepsilon<\min \left(\frac{1}{2}, \delta\right)\right)
$$

or

$$
\rho_{n}=[\log \log (n+2)]^{1 / 2+\iota}
$$

and

$$
\omega_{p}^{(1)}(t, f)=O\left\{\left(\log \frac{1}{t}\right)^{\frac{1}{p}-\frac{3}{2}}\left(\log \log \frac{1}{t}\right)^{-\frac{3}{2}-\delta}\right\} \quad\left(0<\varepsilon<\min \left(\frac{1}{2}, \delta\right)\right)
$$

then the conditions of Theorem I or Theorem II are satisfied, thus the series (1) is $|C, \alpha|(\alpha>1 / p)$ or $|C, 1 / p|$ summable almost everywhere, respectively.

2. We require the following known lemmas :

LEMmA 1, If $f(x) \in L^{p}(1<p<\infty)$, then 


$$
\left\|f(x)-s_{n}(x)\right\|_{p}=O\left\{\omega_{p}^{(2)}\left(\frac{1}{n}, f\right)\right\} .
$$

(See, e. g. [9] p. 339 and [8] p. 226.)

LEMmA 2. (CHOW [3], Theorem I). If $f(x) \in L^{p}(1<p \leqq 2)$ then the series

$$
\sum_{n=1}^{\infty} \frac{\left|\tau_{n}^{\alpha}(x)\right|^{2}}{n}
$$

is convergent for almost all $x$, where $\alpha>1 / p$.

LEMMA 3. If $f(x) \in L^{p}(1<p \leqq 2)$, then the series

$$
\sum_{n=1}^{\infty} \frac{\left|\tau_{n}^{1 / p}(x)\right|^{2}}{n\left[\log (n+1)^{5}\right]^{2-2 / p}}
$$

is convergent for almost all $x$.

This lemma follows from Theorem I of Kozima [4].

LEMMA 4. Let $0<\alpha<1$ and $\left\{\lambda_{n}\right\}$ be a sequence of positive numbers such that $\lambda_{n} \cdot n^{-1}$ is non-increasing and $\Delta \lambda_{n}=\lambda_{n}-\lambda_{n+1}=O\left\{\frac{\lambda_{n}}{n}\right\}$. If the series

$$
\sum_{n=1}^{\infty} \frac{\lambda_{n}\left|\tau_{n}^{\alpha}(x)\right|}{n}
$$

is convergent, then the series $\sum_{n=0}^{\infty} \lambda_{n} A_{n}(x)$ is summable $|C, \alpha|$.

The proof of Lemma 4 runs similarly to that of Lemma 4 of CHOw [2].

LEMMA 5. (KogBENTLIANTZ [5]). If the series $\sum_{n=0}^{\infty} a_{n}$ is summable $|C, \alpha|(\alpha>-1)$, then it is also summable $|C, \alpha+\beta|$ for any $\beta>0$.

3. We prove the following lemmas :

LEMMA 6. Let $f(x) \in L^{p}(1<p \leqq 2)$ and let $\left\{u_{n}\right\}$ be a sequence of positive 
numbers. If

$$
\sum_{n=1}^{\infty} u_{n} \omega_{p}^{(2)}\left(\frac{1}{n}, f\right)<\infty
$$

then $\sum_{n=0}^{\infty} \bar{\lambda}_{n} A_{n}(x)$ is the Fourier series of a function of class $L^{p}$, where $\bar{\lambda}_{0}=1$ and $\bar{\lambda}_{n}=\sum_{k=1}^{n} u_{k}(n=1,2, \cdots)$.

PROOF. Let us denote by $t_{n}(x)$ the $n$-th partial sum of the series $\sum_{n=0}^{\infty} \bar{\lambda}_{n} A_{n}(x)$, i. e., $t_{n}(x)=\sum_{k=0}^{n} \bar{\lambda}_{k} A_{k}(x)$, then

$$
\begin{aligned}
& \left\|t_{n}(x)-f(x)\right\|_{p}=\left\|\bar{\lambda}_{0}\left(A_{0}(x)-f(x)\right)+\sum_{k=1}^{n} \bar{\lambda}_{k} A_{k}(x)\right\|_{p} \\
& =\left\|\sum_{k=0}^{n-1}\left(s_{k}(x)-f(x)\right) \Delta \bar{\lambda}_{k}+\left(s_{n}(x)-f(x)\right) \bar{\lambda}_{n}\right\|_{p} \\
& \leqq \sum_{k=0}^{n-1}\left\|s_{k}(x)-f(x)\right\|_{p}\left|\Delta \bar{\lambda}_{k}\right|+\left\|s_{n}(x)-f(x)\right\|_{p} \bar{\lambda}_{n} \\
& =\sum_{1} .
\end{aligned}
$$

By Lemma 1 and ( 6 ) we have

$$
\begin{aligned}
& \sum_{1} \leqq C_{1} \sum_{k=1}^{n-1} \omega_{p}^{(2)}\left(\frac{1}{k}, f\right) u_{k}+C_{2} \omega_{p}^{(2)}\left(\frac{1}{n}, f\right) \sum_{k=}^{n} " \\
& \leqq C_{1} \sum_{k=1}^{\infty} \omega_{p}^{(2)}\left(\frac{1}{k}, f\right) u_{k}+C_{2} \sum_{k=1}^{n} \omega_{p}^{(2)}\left(\frac{1}{k}, f\right) u_{k} \\
& \leqq C_{3} \sum_{k=1}^{\infty} \omega_{p}^{(2)}\left(\frac{1}{k}, f\right) u_{k}<C .
\end{aligned}
$$

Hence

$$
\left\|t_{n}(x)\right\|_{p} \leqq\left\|t_{n}(x)-f(x)\right\|_{p}+\|f(x)\|_{p}=O(1 .
$$

From this it follows the statement of Lemma 6 . 
LEMMA 7. Let $f(x) \in L^{p}(1<p \leqq 2)$ and let $\left\{v_{n}\right\}$ be a sequence of positive numbers. If

$$
\sum_{n=1}^{\infty} v_{n} \omega_{p}^{(2)}\left(\frac{1}{n}, f\right)[\log (n+1)]^{1-1 / p}<\infty,
$$

then the series $\sum_{n=0}^{\infty} l_{n} A_{n}(x)$ is the Fourier series of a function of class $L^{p}$, where $l_{0}=1$ and $l_{n}=\sum_{k=1}^{n} v_{k}[\log (k+1)]^{1-1 / p}(n=1,2, \cdots)$

PROOF. It runs similarly to the proof of Lemma 6 .

LEMMA 8. Let $f(x) \in L^{p}(1<p \leqq 2)$ and let $\left\{\kappa_{n}\right\}$ be a sequence of positive numbers, such that $\kappa_{n} / n$ is non-increasing and $\Delta \kappa_{n}=O\left(\kappa_{n} / n\right)$. If

$$
\sum_{n=1}^{\infty} \frac{\kappa_{n}^{2}}{n}<\infty
$$

then the series

$$
\sum_{n=0}^{\infty} \kappa_{n} A_{n}(x)
$$

is summable $|C, \alpha|$ almost everywhere, for any $\alpha>1 / p$.

If

$$
\sum_{n=1}^{\infty} \frac{\kappa_{n}^{2}[\log (n+1)]^{2-2 / p}}{n}<\infty
$$

then the series ( 7$)$ is summable $|C, 1 / p|$ almost everywhere.

ProOF. Let $1 / p<\alpha^{\prime}<1$. Applying Schwarz's inequality we have

$$
\sum_{n=1}^{\infty} \frac{\kappa_{n}\left|\tau_{n}^{\alpha^{\prime}}(x)\right|}{n} \leqq\left(\sum_{n=1}^{\infty} \frac{\kappa_{n}^{2}}{n}\right)^{1 / 2}\left(\sum_{n=1}^{\infty} \frac{\left|\tau_{n}^{\alpha^{\prime}}(x)\right|^{2}}{n}\right)^{1 / 2}
$$

From this inequality, by Lemma 2 and Lemma 4, we get that the series ( 7 ) is summable $\left|C, \alpha^{\prime}\right|$ almost everywhere, and by Lemma 5 , we get that the series ( 7) is summable $|C, \alpha|$ almost everywhere, for any $\alpha>1 / p$. 
The proof of the second statement follows the same lines as that of the first statement. Applying Schwarz's inequality we have

$$
\sum_{n=1}^{\infty} \frac{\kappa_{n}\left|\boldsymbol{\tau}_{n}{ }^{1 / p}(x)\right|}{n} \leqq\left(\sum_{n=1}^{\infty} \frac{\left|\tau_{n}^{1 / p}(x)\right|^{2}}{n[\log (n+1)]^{2-2 / p}}\right)^{1 / 2}\left(\sum_{n=1}^{\infty} \frac{\kappa_{n}^{2}[\log (n+1)]^{2-2 / p}}{n}\right)^{1 / 2} .
$$

From this inequality, by Lemma 3 and Lemma 4 we obtain the statement.

4. Proof of Theorem I. Let $\bar{\lambda}_{0}=1$ and $\bar{\lambda}_{n}=\sum_{k=1}^{n} \mu_{k}(n=1,2, \cdots)$. By condition (3) and Lemma 6 we have that $\sum_{n=0}^{\infty} \bar{\lambda}_{n} A_{n}(x)$ is the Fourier series of a function in $L^{p}$.

Let now $\kappa_{n}=\bar{\lambda}_{n}{ }^{-1}(n=0,1, \cdots)$. By condition (2) $\left\{\kappa_{n}\right\}$ satisfies the conditions of Lemma 8 , so we have that the series (1) is summable $|C, \alpha|$ almost everywhere, for any $\alpha>1 / p$, as it was stated.

5. Proof of Theorem II. Let $v_{n}=\frac{\rho_{n}}{n \sqrt{\log (n+1)}}(n=1,2, \cdots)$. By condition ( 5 ) and Lemma 7 we have that $\sum_{n=0}^{\infty} l_{n} A_{n}(x)$ is the Fourier series of a function in $L^{p}$, where $l_{0}=1$ and $l_{n}=\sum_{k=1}^{n} \frac{\rho_{k}}{k[\log (k+1)]^{1 / p-1 / 2}}$.

Let now $\kappa_{n}=l_{n}^{-1}(n=0,1, \cdots)$. Since

$$
\begin{aligned}
& \Delta \kappa_{n}=\kappa_{n}-\kappa_{n+1}=\frac{1}{l_{n}}-\frac{1}{l_{n+1}} \\
& =\frac{\rho_{n+1}}{(n+1)[\log (n+2)]^{1 / p-1 / 2} l_{n} \cdot l_{n+1}} \\
& \leqq \frac{\rho_{n+1}}{(n+1)[\log (n+2)]^{1 / p-1 / 2} \cdot l_{n} \sum_{k=1}^{n+1} \frac{\rho_{k}[\log (k+1)]^{1-1 p}}{\sqrt{\log (k+1) \cdot k}}} \\
& \leqq \frac{\rho_{n+1}}{(n+1)[\log (n+2)]^{1 / p-1 / 2} \cdot l_{n} \frac{\rho_{n+1}[\log (n+2)]^{1-1 / p}}{\sqrt{\log (n+2)}}} \\
& =\frac{1}{(n+1) l_{n}}<\frac{\kappa_{n}}{n}
\end{aligned}
$$

and since on the other hand 


$$
\begin{aligned}
& \sum_{n=1}^{\infty} \kappa_{n}^{2}\left[\log \frac{(n+1)]^{2-2 / p}}{n}=\sum_{n=1}^{\infty} \frac{[\log (n+1)]^{2-2 / p}}{n l_{n}^{2}}\right. \\
& \leqq \sum_{n=1}^{\infty} \frac{[\log (n+1)]^{2-2 / p}}{n\left(\frac{\rho_{n}}{\left.\sqrt{\log (n+1)} \sum_{k=1}^{n} \frac{[\log (k+1)]^{1-1 / p}}{k}\right)^{2}}\right.} \\
& \leqq C \sum_{n=1}^{\infty} \frac{[\log (n+1)]^{2-2 / p}}{n \rho_{n}^{2}[\log (\mathrm{n}+1)]^{3-2 / p}} \\
& =C \sum_{n=1}^{\infty} \frac{1}{n \rho_{n}^{2} \log (n+1)}<\infty
\end{aligned}
$$

the sequence $\left\{\kappa_{n}\right\}$ satisfies the conditions of Lemma 8. By using of Lemma 8 we have that the series $(1)$ is summable $|C, 1 / p|$ almost everywhere.

This completes the proof of Theorem II.

\section{REFERENCES}

[1] H. C. CHOw, Some new criteria for the absolute summability of a Fourier series and its conjugate series, J. London Math. Soc., 30(1955), 133-142.

[2] H. C. CHOW, Theorems on power series and Fourier series, Proc. London Math. Soc., 1(1951), 206-216.

[3] H.C.CHOW, An extension of a theorem of Zygmund and its application, J. London Math. Soc., 29(1954), 189-197.

[4] M. KozIMA, On the absolute summability factors of power series, Tôhoku Math. J., 18(1966), 60-70.

[5] E. Kogbentliantz, Sur les séries absolument sommables par la méthode des moyennes arithmétiques, Bull. Sci. Math., 49(1925), 234-256.

[6] L. LEINDLER, Über verschiedene Konvergenzarten trigonometrischer Reihen III, Acta Sci. Math., 27(1966), 205-215.

[ 7 ] G. SuNOUCHI, Some criteria for the absolute summability of a Fourier series, Tôhoku Math. J., 19(1967), 311-314.

[8] S. B. STECKIN, On the order of the best approximations of continuous functions, Izv. Akad. Nauk SSSR., 15(1951), 219-242.

[9] A. F. TIMAN, Theory of approximation of functions of a real variable, Moscow, 1960.

DEPARTMENT OF MATHEMATICS

UNIVERSITY OF SZEGED

HUNGARY 